\title{
Efficacy of Noninvasive Positive Pressure Ventilation for Improving the Respiratory Function, Use of Accessory Respiratory Muscles, Quality of Sleep and Nutrition of Cystic Fibrosis Patients
}

\author{
Maryam Hassanzad, ${ }^{1}$ Shahram Kharabian Masouleh,,, ${ }^{, *}$ Sabereh Tashayoie Nejad, ${ }^{1}$ Saba Karimzadeh, ${ }^{3}$ \\ and Ali Akbar Velayati ${ }^{4,5}$ \\ ${ }^{1}$ M.D. Pediatric Respiratory Diseases Research Center, National Research Institute of Tuberculosis and Lung Diseases (NRITLD), Shahid Beheshti University of Medical Sciences, \\ Tehran, IR Iran \\ ${ }^{2}$ M.D. Chronic Respiratory Disease Research Center, National Research Institute of Tuberculosis and Lung Diseases (NRITLD), Shahid Beheshti University of Medical Sciences, \\ Tehran, IR Iran \\ ${ }^{3}$ Msc. Chronic Respiratory Disease Research Center, National Research Institute of Tuberculosis and Lung Diseases (NRITLD), Shahid Beheshti University of Medical Sciences, \\ Tehran, IR Iran \\ ${ }^{4}$ M.D. Mycobacteriology Research Center, National Research Institute of Tuberculosis and Lung Diseases (NRITLD), Shahid Beheshti University of Medical Sciences, Tehran, IR \\ Iran \\ ${ }^{5}$ Clinical Tuberculosis and Epidemiology Research Center, National Research Institute of Tuberculosis and Lung Diseases (NRITLD), Shahid Beheshti University of Medical \\ Sciences, Tehran, IR Iran \\ "Corresponding author: Shahram Kharabian Masouleh, Masih Daneshvari Hospital, Darabad Avenue, Shahid Bahonar roundabout, P,O, Box: 1956944413, Tehran, IR Iran. Tel: \\ +98-9122002452, E-mail: mar_hassanzad@yahoo.com
}

Received 2017 June 07; Accepted 2017 June 30.

\begin{abstract}
Background: Respiratory failure secondary to recurrent respiratory infections is the most common cause of death in Cystic fibrosis (CF).

Objectives: To assess the efficacy of noninvasive positive pressure ventilation (NIPPV) on respiratory function, use of accessory respiratory muscles, ease of physiotherapy for respiratory clearance, quality of sleep and nutritional status in CF patients with respiratory failure.

Methods: All CF patients admitted to the pediatric department of Masih Daneshvari hospital, Tehran from March 2015 to January 2016 were studied. The inclusion criteria were clinical evidence of respiratory distress and signs of respiratory acidosis. The exclusion criteria were nausea and vomiting, not tolerating NIPPV, need for intubation, pneumothorax, presence of giant bullous emphysema or decreased level of consciousness. Baseline spirometry and venous blood gas (VBG) was obtained before and after NIPPV. A simple questionnaire was filled out for the nutritional status, sleep quality, ease of physiotherapy for airway clearance and the volume of secretions after NIPPV compared to baseline. Paired samples t-test and Wilcoxon Signed Rank test in SPSS version 16 were used for the before and after comparison of numerical and ordinal variables, respectively.

Results: Out of a total of 53 CF hospitalized patients 17 met the inclusion criteria. There were 10 (58.8\%) males and 7 (41.1\%) females with a mean age of $11 \pm 4.57$ (10 - 25) years, mean weight of $31.85 \pm 10.11$ (13 - 48) $\mathrm{kg}$ and mean BMI of $14.16 \pm 2.71$ (5.9-18). The mean Saturation of peripheral oxygen $\left(\mathrm{SPO}_{2}\right)$, respiratory rate $(\mathrm{RR})$ and mean partial pressure of $\mathrm{CO}_{2}\left(\mathrm{PCO}_{2}\right)$ of patients before the intervention were $75 \pm 13.25 \%$ (52 - 90\%), $32 \pm 11.17$ (22 - 55) $\mathrm{mmHg}$ and $55.20 \pm 17.62$ (30.3 - 108), mmHg respectively. A significant difference was noted after the intervention in $\mathrm{SPO}_{2}$, venous $\mathrm{PCO}_{2}$ and RR compared to baseline $(\mathrm{P}<0.05)$. The change in sleep quality $(\mathrm{P}=0.001)$, nutritional status $(\mathrm{P}=0.001)$ and ease of physiotherapy for respiratory clearance $(\mathrm{P}=0.008)$ after NIPPV was statistically significant while the change in use of accessory respiratory muscles $(\mathrm{P}=0.785)$ and the volume of secretions $(\mathrm{P}=0.1)$ was not significant.

Conclusions: NIPPV in CF patients with respiratory failure can improve the respiratory function, sleep quality and nutritional status of patients. Also, NIPPV enhances airway clearance during respiratory physiotherapy.
\end{abstract}

Keywords: Cystic Fibrosis, Noninvasive Positive Pressure Ventilation

\section{Background}

Cystic fibrosis is a relatively common disease with a prevalence of 1/2500 live births in Caucasian race. Despite the involvement of various organs, respiratory infections and respiratory failure are the most common causes of death in CF patients (1). Comprehensive studies on the prevalence of CF in Iran are not available (2-5); however, its prevalence in the Middle East has been reported to be $1 / 2850$ to $1 / 5800(6,7)$. Respiratory failure in CF patients is characterized by impairments in gas exchange and arterial blood gases (ABG) (8). Moreover, airway obstruction due to progressive inflammation and progressive sputum production results in dyspnea, airway hyperinflation, 
impaired ventilation and perfusion (VQ mismatch), respiratory distress and use of accessory respiratory muscles, which gradually decrease maximum inspiratory pressure (MIP) and maximum expiratory pressure (MEP) and cause respiratory muscle fatigue and impaired blood gas exchange at the level of alveoli and consequent blood gas impairment $(8,9)$. Long term hypoxia gradually increases the pulmonary artery pressure and leads to cor pulmonale (10). At present, mask NIPPV is used to assist respiration of patients with acute respiratory failure (11). In this technique, patient's respiration is assisted by use of devices similar to ventilators with predetermined pressures and volumes during inhalation and exhalation via different oral and nasal masks. Hodson et al. (12) and Pipr et al. (13) showed that NIPPV reversed hypercapnia and decreased respiratory muscle fatigue by increasing alveolar ventilation. The suggested mechanism for the efficacy of NIPPV is increased alveolar ventilation and decreased hypoventilation and respiratory work $(11,14)$. Also, NIPPV enhances the clearance of pulmonary secretions in CF patients; however, its exact mechanism has yet to be fully understood (10). In general, use of NIPPV has been documented in respiratory physiotherapy guidelines for CF patients especially when the respiratory muscle weakness is due to fatigue (15). Considering the advantages of using NIPPV in pulmonary patients and high prevalence of respiratory failure in CF patients, NIPPV may be helpful in these patients (16).

\section{Objectives}

This study sought to assess the efficacy of NIPPV in decreasing pulmonary secretions and improving the respiratory function, quality of sleep and nutrition of CF patients in a tertiary referral center for respiratory diseases.

\section{Methods}

All CF patients presenting to Masih Daneshvari hospital in Tehran from March 2015 to January 2016 were evaluated. Parents or legal guardians of patients signed written informed consent forms prior to the inclusion of patients in the study. The inclusion criteria were CF patients with the criteria of acute or chronic respiratory failure including clinical evidence of respiratory distress (tachypnea and use of accessory respiratory muscles), evidence of respiratory acidosis including a $\mathrm{pH}<7.35$ or $\mathrm{PCO}_{2} \geq 45 \mathrm{mmHg}$ or clinical evidence of long-term hypoxemia or hypercapnia. Patients with the following signs/symptoms were excluded from the study:

1. Nausea and vomiting

2. Not tolerating NIPPV
3. Requiring intubation based on consultation with an anesthesiologist

4. Occurrence of pneumothorax

5. Giant bullous emphysema

6. Decreased level of consciousness

All patients were subjected to baseline spirometry according to the American Thoracic Society guidelines and their forced vital capacity (FVC), forced expiratory volume in one second (FEV1) and FEV1/FVC were recorded. Also, since frequent blood gas monitoring was required, VBG was obtained instead of ABG to decrease patients' pain and discomfort, and $\mathrm{pH}$ and $\mathrm{PCO}_{2}$ were also recorded.

Patients' medical history was taken and a simple questionnaire was filled out to assess the use of accessory respiratory muscles, sleep quality and nutritional status of patients as reported by patients themselves or their companions and categorized as poor, moderate and good. The arterial oxygen saturation rate was recorded using a contact pulse oxymeter at rest.

For NIPPV, a standard oronasal mask with a suitable size for each patient was used. A Resmed S9 VPAP Auto machine with Bi level positive airway pressure (BIPAP) option was used by an experienced nurse under the supervision of a pediatric pulmonologist. The inhalation and exhalation pressures were adjusted according to the published guidelines, patient's comfort and blood gas status (13). The NIPPV was performed overnight, during sleep and four times a day, each time for two hours under supervision of a nurse. The NIPPV was performed for 72 hours as such. The mask was removed from the face for respiratory physiotherapy or eating. The same techniques including positional drainage, stimulating an effective cough and massage of the chest wall were used for all patients. The ease of physiotherapy for airway clearance and the volume of secretions were also recorded by the attending physician and physiotherapist. The data were analyzed using SPSS version 16. Paired samples t-test was used to analyze the changes in $\mathrm{RR}, \mathrm{PCO}_{2}$ and $\mathrm{SPO}_{2}$ after the intervention compared to baseline. Changes in ordinal variables including the use of accessory respiratory muscles, sleep quality, nutritional status, volume of secretions and ease of physiotherapy for respiratory clearance were analyzed after the intervention compared to baseline using Wilcoxon Signed Rank test.

\section{Results}

During the nine-month period, from $53 \mathrm{CF}$ patients hospitalized in the Pediatric Ward of Masih Daneshvari Hospital; 17 (32.07\%) met the inclusion criteria and thirty six patients (67.9\%) excluded due to absence of respiratory distress $(n=36)$, respiratory acidosis $(n=36)$, hypoxia $(n=$ 
24 ) or long-term hypercapnia $(n=32)$. There were 10 males (58.8\%) and seven females (41.1\%). The mean age of patients was $17.11 \pm 4.15$ years (range 10 - 25 years). The mean weight of patients was $31.85 \pm 10.11 \mathrm{~kg}$ (range 13 - $48 \mathrm{~kg}$ ). The mean height of patients was $147.18 \pm 15.94 \mathrm{~cm}$ (range $117-165 \mathrm{~cm}$ ). The mean BMI was $14.16 \pm 2.71$ (5.9-18). The mean $\mathrm{SPO}_{2}$, the mean RR and the mean $\mathrm{PCO}_{2}$ of patients was $75 \pm 13.25$ (52 $90) \%, 32 \pm 11.17(22-55) \mathrm{mmHg}$ and $55.20 \pm 17.62(30.3-108)$ $\mathrm{mmHg}$, respectively.

Normal distribution of data was checked by Kolmogorov-Smirnov test $(\mathrm{P}>0.05)$. The mean pressure used by BIPAP machine in patients was $10.4 \pm 3.21$ and $6.6 \pm 3.4 \mathrm{Cm} \mathrm{H}_{2} \mathrm{O}$ for IPAP and EPAP, respectively.

The RR, $\mathrm{PCO}_{2}$ and $\mathrm{SPO}_{2}$ values before and after the intervention and their comparison with paired t-test are presented in Table1. As seen in the the the change in $\mathrm{SPO}_{2}, \mathrm{PCO}_{2}$ and RR was statistically significant after the intervention compared to baseline $(\mathrm{P}<0.02)$. Also, the change in sleep quality $(\mathrm{P}=0.001)$, nutritional status $(\mathrm{P}=0.001)$ and ease of physiotherapy for respiratory clearance $(\mathrm{P}=0.008)$ after NIPPV was statistically significant while the change in use of accessory respiratory muscles $(\mathrm{P}=0.785)$ and the volume of secretions $(\mathrm{P}=0.1)$ was not significant.

Table 1. The RR, $\mathrm{PCO}_{2}$ and $\mathrm{SPO}_{2}$ Values Before and After NIPPV

\begin{tabular}{lccc}
\hline Variable & Before & After & PValue \\
\hline $\mathbf{S P O}_{2}$ & $75 \pm 13.25$ & $91 \pm 3.37$ & 0.000 \\
$\mathbf{P C O}_{2}$ & $55.2 \pm 17.6$ & $46.2 \pm 13.01$ & 0.01 \\
$\mathbf{R R}$ & $32.3 \pm 11.1$ & $22.5 \pm 2.3$ & 0.004 \\
\hline
\end{tabular}

\section{Discussion}

The purpose of this study was to evaluate the efficacy of NIPPV as a relatively novel intervention in decreasing pulmonary secretions and improving the respiratory function, quality of sleep and nutrition of CF patients in a referral center for respiratory diseases. The study was addressing the following questions:

1. Is NIPPV able to decrease the RR, $\mathrm{PCO}_{2}$ and use of accessory respiratory muscles as the hallmarks of respiratory distress and failure?

2. Does NIPPV increase the $\mathrm{SPO}_{2}$ significantly?

3. Does the use of NIPPV increase the quality of sleep and nutritional status dramatically?

4. Is the airway clearance after NIPPV easier by the way of chest physical therapy?

In the current study, BIPAP option was used for NIPPV and the IPAP and EPAP pressures were adjusted based on patient's tolerance and titration of VBG, and were in agreement with previous studies (17). Our results showed that use of NIPPV in CF patients significantly increased $\mathrm{SPO}_{2}(\mathrm{P}$ $<0.05$ ) and caused a significant reduction in $\mathrm{PCO}_{2}$ and $\mathrm{RR}$ of patients $(\mathrm{P}<0.05)$. Also, our results showed that use of NIPPV significantly improved the sleep quality, nutritional status and ease of respiratory clearance by physiotherapy $(\mathrm{P}<0.05)$; although the effect of NIPPV on use of accessory respiratory muscles and the objective volume of secretions was not significant $(\mathrm{P}>0.05)$.

Despite the availability of several studies on the use of NIPPV in different respiratory diseases, no definite consensus has been reached on selection of the mode of machine or inhalation or exhalation pressures in NIPPV (18). This is also true for use of NIPPV for respiratory failure in children. In a review study by Teague et al. (17) on the indications for use of NIPPV in pediatric medicine, the agreed values for inspiratory positive airway pressure (IPAP) and expiratory positive airway pressure (EPAP) in CF patients were reported to be $8-18 \mathrm{Cm} \mathrm{H}_{2} \mathrm{O}$ and $4-10 \mathrm{Cm} \mathrm{H}_{2} \mathrm{O}$, respectively. Flight et al in their nine-year study on hospitalized patients with $\mathrm{CF}$, who had received NIPPV, reported that the lowest IPAP pressure was $2.7 \mathrm{Cm} \mathrm{H}_{2} \mathrm{O}$ without applying EPAP; in other words, continues positive airway pressure (CPAP) was used (19). They showed that use of CPAP caused significant improvements in CF patients.

The effect of NIPPV on $\mathrm{SPO}_{2}$ of CF patients has been evaluated in many previous studies. Kofler et al. reported increased $\mathrm{SPO}_{2}$ following NIPPV alone compared to the group receiving respiratory physiotherapy (20). In their study, level of $\mathrm{SPO}_{2}$ in NIPPV group was significantly higher than that in the group receiving respiratory physiotherapy including respiratory exercises and chest wall massage. Moreover, Holland et al. compared the efficacy of NIPPV and standard pharmaceutical therapy plus physiotherapy and reported significantly higher $\mathrm{SPO}_{2}$ values in NIPPV group $(P=0.001)(21)$. However, these findings were in contrast to the results of Placidi et al. who compared NIPPV with respiratory physiotherapy including stimulation of coughing and found no significant difference (1). Studies on the efficacy of NIPPV for decreasing pulmonary secretions are mainly inconclusive. Fauroux et al. and Holland et al. found no significant difference in respiratory clearance between the NIPPV and respiratory physiotherapy by stimulation of cough $(21,22)$. However, in the study by Fauroux et al. the fatigue of patients after clearance in NIPPV group was less than that in patients who only received respiratory physiotherapy (22). In a recent study by Rodriguez Horta the effect of NIPPV compared to conventional chest physical therapy and positive expiratory pressure (PEP) on increasing the rate of airway clearance in CF was significant (23). They studied 32 subjects with 
the mean age of 31 and used BIPAP mode for NIPPV [IPAP $(10-20) \mathrm{Cm} \mathrm{H}_{2} \mathrm{O}$. Thy reported the significant reduction in lung clearance index (LCI) in NIPPV group.

The efficacy of NIPPV for improving sleep quality of CF patients has been evaluated in a few studies. Young et al evaluated the effect of non-invasive ventilation on sleep quality of eight patients with CF using a sleep questionnaire (24). They did not find a significant difference in sleep quality or PCO2 of patients when awake between the two groups of NIPPV and nasal oxygenation alone; however, significant differences were noted in nocturnal $\mathrm{PCO}_{2}$, quality of life and nocturnal dyspnea. The findings of Young et al are in contrast to our results. This controversy may be attributed to the difference in the quality of questionnaires used in the two studies. Our questionnaire was qualitative, ordinal and self-reported by patients. Moreover, we did not have a control group. The efficacy of NIPPV was previously evaluated by Young et al. and Milross et al. $(22,25)$. The study by Young et al was conducted in room air. Milross et al. used NIPPV combined with oxygen. In both studies, arterial $\mathrm{PCO}_{2}$ significantly decreased compared to the group receiving oxygen alone; this finding was in agreement with our result and was due to the improved respiratory function by NIPPV. Also, studies performing sleep tests such as polysomnography have reported significant improvement of sleep quality in patients receiving NIPPV (25).

Assessment of the effect of NIPPV on use of accessory respiratory muscles was strength of our study since this variable has not been evaluated before in CF patients receiving NIPPV. The results showed that despite the significant improvement in objective indices of respiratory function including reduction in arterial $\mathrm{PCO}_{2}$ and increased arterial $\mathrm{SPO}_{2}$, use of accessory respiratory muscles by patients receiving NIPPV was still significantly high. Considering the chronicity of $\mathrm{CF}$ and long-term changes in respiratory mechanics of these patients, correction of their respiratory habits and use of diaphragm instead of accessory respiratory muscles require long-term respiratory physiotherapy exercises in addition to the use of NIPPV to improve respiratory indices.

Also, considering the long-term course of $\mathrm{CF}$, future studies with larger sample sizes are required to assess the efficacy of NIPPV in decreasing the need for hospital admissions of CF patients.

\subsection{Limitations}

Although we tried to carefully prepare the study, we are still aware of its limitations. First of all, the sample size is really small. It should be mentioned that this report is a part of a greater study and we hope to gather more samples in the future. Second, in this study we didn't have any control group and the results are compared to the baseline. It seems that introducing a control group in which the mask of NIPPV is placed but the device turned off would be of value. Third, since the questionnaire was designed to measure sleep quality in our study, it seems not to provide enough evidence compared to polysomnography data as an objective measurements for sleep study.

\subsection{Conclusions}

Use of NIPPV in CF patients with acute or chronic respiratory failure can improve their respiratory function, nutritional status and sleep quality. Also, this method significantly enhances respiratory clearance during respiratory physiotherapy. Long-term studies on a larger group of patients are required to assess the effect of NIPPV on survival of CF patients.

\section{References}

1. Placidi G, Cornacchia M, Polese G, Zanolla L, Assael BM, Braggion C. Chest physiotherapy with positive airway pressure: a pilot study of short-term effects on sputum clearance in patients with cystic fibrosis and severe airway obstruction. Respir Care. 2006;51(10):1145-53. [PubMed: 17005060].

2. Baghaie N, Kalilzadeh S, Hassanzad M, Parsanejad N, Velayati A. Determination of mortality from cystic fibrosis. Pneumologia. 2009;59(3):170-3.

3. Khalilzadeh S, Kahkouee S, Hassanzad M, Parsanejad N, Baghaie N, Bloorsa MR. The correlation of brody high resolution computed tomography scoring system with clinical status and pulmonary function test in patients with cystic fibrosis. Iran J Med Sci. 2011;36(1):18-23. [PubMed: 23365473].

4. Khalilzadeh S, Hassanzad M, Baghaie N. Shwachman score in clinical evaluation of cystic fibrosis. J Compreh Pediatr. 2012;4(1):82-5.

5. Motamed F, Moayednia M, Moayednia N, Najafi Sani M, Farahmand F, Khodadad A, et al. Clinical Presentations of Cystic Fibrosis in Iranian Children. Iran J Pediatr. 2015;25(2):e255. doi: 10.5812/ijp.255. [PubMed: 26195997].

6. Al-Mahroos F. Cystic fibrosis in bahrain incidence, phenotype, and outcome. J Trop Pediatr. 1998;44(1):35-9. [PubMed: 9538604].

7. Rawashdeh M, Manal H. Cystic fibrosis in Arabs: a prototype from Jordan. Ann Trop Paediatr. 2000;20(4):283-6. [PubMed: 11219165].

8. Noone PG. Non-invasive ventilation for the treatment of hypercapnic respiratory failure in cystic fibrosis. Thorax. 2008;63(1):5-7. doi: 10.1136/thx.2007.086710. [PubMed: 18156565].

9. Madden BP, Kariyawasam H, Siddiqi AJ, Machin A, Pryor JA, Hodson ME. Noninvasive ventilation in cystic fibrosis patients with acute or chronic respiratory failure. Eur Respir J. 2002;19(2):310-3. [PubMed: 11866011].

10. Eckles M, Anderson P, editors. Cor pulmonale in cystic fibrosis. Seminars in respiratory and critical care medicine. 2003; New York. pp. 323-30.

11. Moran F, Bradley JM, Piper AJ. Non-invasive ventilation for cys tic fibrosis. Cochrane Database Syst Rev. 2017;2:CD002769. doi: 10.1002/14651858.CD002769.pub5. [PubMed: 28218802].

12. Hodson ME, Madden BP, Steven MH, Tsang VT, Yacoub MH. Noninvasive mechanical ventilation for cystic fibrosis patients-a potential bridge to transplantation. Eur Respir J. 1991;4(5):524-7. [PubMed: 1936222]. 
13. Piper AJ, Parker S, Torzillo PJ, Sullivan CE, Bye PT. Nocturnal nasal IPPV stabilizes patients with cystic fibrosis and hypercapnic respiratory failure. Chest. 1992;102(3):846-50. [PubMed: 1516413].

14. Moran F, Bradley J. Non-invasive ventilation for cystic fibrosis. Cochrane Database Syst Rev. 2003(2):CD002769. doi: 10.1002/14651858.CD002769. [PubMed: 12804435].

15. Bott J, Blumenthal S, Buxton M, Ellum S, Falconer C, Garrod R, et al. Guidelines for the physiotherapy management of the adult, medical, spontaneously breathing patient. Thorax. 2009;64(Suppl 1):i1-i52.

16. Oktem S, Ersu R, Uyan ZS, Cakir E, Karakoc F, Karadag B, et al. Home ventilation for children with chronic respiratory failure in Istanbul. Respiration. 2008;76(1):76-81. doi: 10.1159/000110801. [PubMed: 17984626].

17. Teague WG. Non-invasive positive pressure ventilation: current status in paediatric patients. Paediatr Respir Rev. 2005;6(1):52-60. doi: 10.1016/j.prrv.2004.11.014. [PubMed:15698817].

18. Holland AE, Button BM, International Physiotherapy Group for Cystic Fibrosis AC. Physiotherapy for cystic fibrosis in Australia: knowledge and acceptance of the Consensus Statement recommendations. Respirology. 2013;18(4):652-6. doi: 10.1111/resp.12032. [PubMed: 23240864].

19. Flight WG, Shaw J, Johnson S, Webb AK, Jones AM, Bentley AM, et al. Long-term non-invasive ventilation in cystic fibrosis - ex- perience over two decades. J Cyst Fibros. 2012;11(3):187-92. doi: 10.1016/j.jcf.2011.11.006. [PubMed: 22177738].

20. Kofler A, Carlesi A, Cutrera R. BiPAP versus PEP as chest physiotherapy in patients with cystic fibrosis. Pediatr Pulmonol. 1998;26(S17):344.

21. Holland AE, Denehy L, Ntoumenopoulos G, Naughton MT, Wilson JW. Non-invasive ventilation assists chest physiotherapy in adults with acute exacerbations of cystic fibrosis. Thorax. 2003;58(10):8804. [PubMed: 14514944].

22. Fauroux B, Boule M, Lofaso F, Zerah F, Clement A, Harf A, et al. Chest physiotherapy in cystic fibrosis: improved tolerance with nasal pressure support ventilation. Pediatrics. 1999;103(3):E32. [PubMed: 10049988].

23. Rodriguez Hortal MC, Nygren-Bonnier M, Hjelte L. Non-invasive Ventilation as Airway Clearance Technique in Cystic Fibrosis. Physiother Res Int. 2017;22(3) doi: 10.1002/pri.1667. [PubMed: 26929396].

24. Young AC, Wilson JW, Kotsimbos TC, Naughton MT. Randomised placebo controlled trial of non-invasive ventilation for hypercapnia in cystic fibrosis. Thorax. 2008;63(1):72-7. doi: 10.1136/thx.2007.082602. [PubMed: 17675317].

25. Milross MA, Piper AJ, Norman M, Willson GN, Grunstein RR, Sullivan $\mathrm{CE}$, et al. Night-to-night variability in sleep in cystic fibrosis. Sleep Med. 2002;3(3):213-9. [PubMed: 14592210]. 